\title{
The Interacting Binary Be Star HR 2142
}

\author{
Geraldine J. Peters \\ Space Sciences Center, University of Southern California, Los Angeles, \\ CA 90089-1341, USA
}

\section{Douglas R. Gies}

CHARA, Department of Physics \& Astronomy, Georgia State University, Atlanta, GA 30303-3083, USA

\begin{abstract}
Current information on the nature of the HR 2142 system is presented. The circumstellar material (CSM) including the mass flow toward and away from the mass-gaining B star has been studied from 100 IUE SWP HIRES images obtained from 1979-95 and ground-based CCD data from KPNO acquired between 1985-2001. The strength and velocity behavior of the infall components to the UV Si II lines seen during the primary shell phase (PSP) from $\phi \sim 0.70-0.98$ resembles that observed in conventional Algol systems, but the inferred mass infall rate is too small to account for the massive $\mathrm{H} \alpha$-emitting disk about the B primary. The cause for the mass outflow observed during the secondary shell phase (SSP) remains unknown, but a localized plasma that has been identified from phase-dependent variations in the emission component to He I $\lambda 6678$ may be part of the SSP phenomenon. Although the secondary remains undetected in the optical/IR spectral regions, UV data currently do not yield strong support for the presence of an O subdwarf as in the $\phi$ Per system. HR 2142 may represent and earlier evolutionary stage of $\phi$ Per and a possibly similar Be binary 59 Cyg.
\end{abstract}

\section{Introduction and History}

The evolutionary state of HR 2142 (HD 41335, V696 Mon, B1.5 IV-Vnne, $v \sin i=350 \mathrm{~km} \mathrm{~s}^{-1}, P=80 \mathrm{~d} 860$ ) has been a subject for debate every since the star was identified as an interacting binary in the early 1970s. In the optical spectral region HR 2142 has all the appearances of a "classical" Be star, but it is unusual in that transient Balmer shell lines from the circumstellar material (CSM) are observed cyclically every $80 \mathrm{~d} 86$ (Peters 1971, 1972). This 'shell' phase is observed in the optical and UV and has two components, each of which displays a characteristic strength and velocity behavior. In the optical spectral region, the first segment (duration of 5-8 days) is characterized by Balmer shell features that are red-shifted relative to the photospheric lines. They peak in strength $\sim 5 \mathrm{~d}$ after they are first detected, then disappear $\sim 3 \mathrm{~d}$ afterwards. The velocities steadily decrease from $\sim 100$ to $20 \mathrm{~km} \mathrm{~s}^{-1}$ during this period. About 2-3 $\mathrm{d}$ after their disappearance, the shell lines reappear at their previous max- 
imum strength for $\sim 3 \mathrm{~d}$, but display negative radial velocities. Following the terminology adopted in the 1970s, the first segment of the two-component shell phase is called the primary shell phase (PSP) while the second part is referred to as the secondary shell phase (SSP).

The binary nature of HR 2142 was eventually confirmed (Peters 1983). The periodic shell activity is caused by an asymmetrical distribution of circumstellar plasma in the system. Every orbital cycle our line-of-sight to the mass gainer sweeps through infalling and counterstreaming material. If the mass of the $\mathrm{Be}$ star is representative of its spectral type (about 10-11 $\mathrm{M}_{\odot}$, Harmanec 1988), and the inclination of the system is $\sim 75^{\circ}$, the semimajor axis for the system is $0.81-0.84 \mathrm{AU}$. The strong Balmer emission ( $\mathrm{H} \alpha$ is $\sim 4.0 I_{\text {cont }}$ ) suggests the presence of a massive circumstellar (CS) disk that probably extends out to the limit of the Roche surface of the primary (Peters 1976). Because of the very conspicuous mass flow toward the B star, it is reasonable to assume that the secondary star fills its critical Roche surface, but its spectrum has never been observed. Waters et al. (1991) suggested that the secondary might be a helium subdwarf, as they failed to find spectral signatures of a cool companion in the IR region. A review of the state of our knowledge of this system as of October 2000 , including a discussion about the possible nature of the still undetected secondary star can be found in Peters (2001a).

From 1979-95 HR 2142 was semi-regularly observed with the HIRES cameras on the IUE spacecraft. About 100 SWP HIRES images were acquired that form a unique database for investigating the mass flow in the system and its long-term behavior. The IUE observations also allow us to put limits on the UV brightness of the undetected secondary. Complementary optical CCD data in the region of $\mathrm{H} \alpha$ and $\mathrm{He} \mathrm{I} \lambda 6678$ acquired with the Coudé Feed Telescope at Kitt Peak National Observatory (KPNO) from 1985-2001 are useful for studying the asymmetry and temporal variability of the CS disk. Some recent results from the analysis of the UV and optical data, including the search for the elusive secondary are summarized in this paper.

\section{The Mass Infall/Outflow Revealed by the UV Spectra}

Mass infall/outflow is observed in many UV features observed with IUE, but perhaps the most useful are the resonance doublets of SiII. These lines are useful because their $f$-values show an enormous range, from $1.2(\lambda \lambda 1260,1264)$ to $0.006(\lambda \lambda 1808,1817)$, and in each pair one member of the doublet lies well enough above the ground state $\left(287 \mathrm{~cm}^{-1}\right)$ that an interstellar component does not contaminate it. Column densities from unsaturated lines are by far the most reliable. From the Si II doublets alone one can reliably determine line-ofsight column densities that differ by more than a factor of 200 . In HR 2142 unsaturated lines from one or more of the SiII doublets are seen at all phases. In one extreme, we observe Si II $\lambda 1817$ absorption as we view the B star through the densest portion of the infalling material $(\phi=0.96)$, whereas in the other we barely detect an enhancement in $\lambda 1264$ as our line-of-sight just intercepts infalling the plasma.

The nature of the infalling/outflowing regions can be seen from the behavior of the $\lambda 1264$ line (Fig. 1a). Absorption from the CSM can be seen as early as 

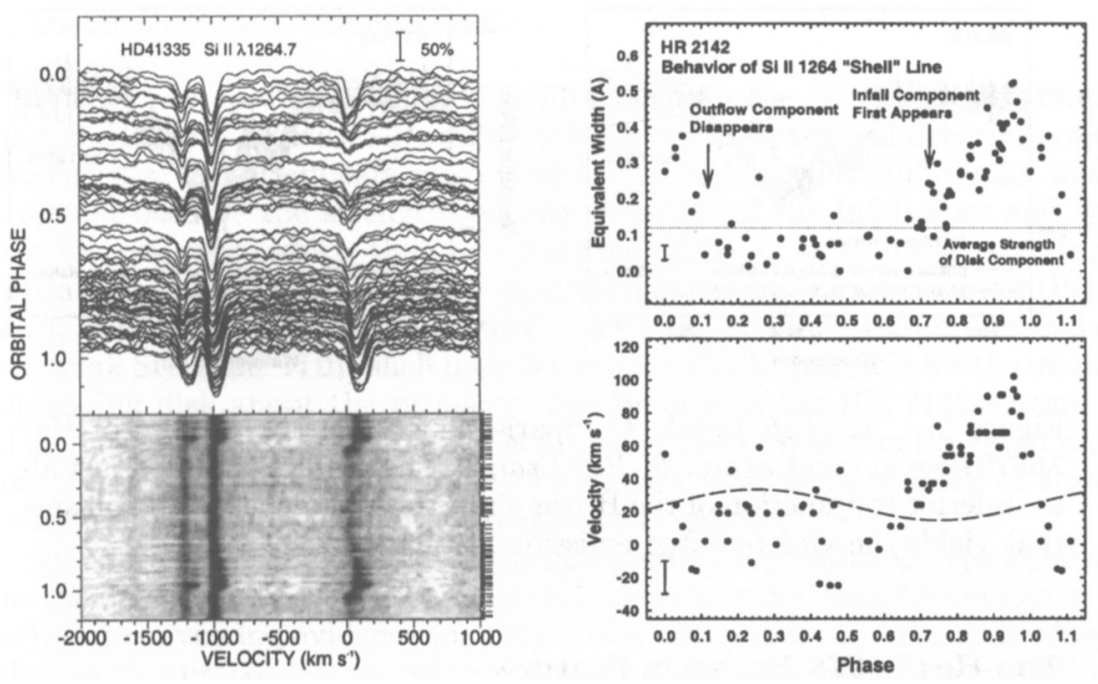

Figure 1. a. Left panel: The behavior of the Si II $\lambda \lambda 1260,1264$ doublet versus phase. The individual profiles and a grayscale (darkness increases with line absorption) display are shown. The arrows to the right indicate the locations of the individual images shown above. The velocities are relative to the $\lambda 1264$ component and are not corrected for binary motion. Interstellar components are present in S II $\lambda 1259$ and SiII $\lambda 1260$. A weak disk component is seen outside of the 'shell' phases. $b$. Right panel: The equivalent width and centroid velocity of the non-photospheric absorption component in $\mathrm{Si}$ II $\lambda 1264$ versus phase. Arrows point to the locations of the disappearance/appearance of the outflow/infall features. The dashed line in the lower panel shows the radial velocity curve for the primary from Peters (1983).

phase 0.7 . The velocity of the feature relative to the photosphere ranges from $0-$ $75 \mathrm{~km} \mathrm{~s}^{-1}$ between phases 0.70 and 0.97 . The strength rapidly declines and the velocity approaches the photospheric value as the stars come into conjunction. The line strength then rapidly increases and the feature becomes violet-shifted as the SSP commences. The behavior of Si II $\lambda 1264$ is shown in Fig. 1b. Note that the outflow component observed during the SSP is seen between phases $0.02-0.09$ with a maximum strength at phase 0.05 , where the Balmer shell lines disappear. There is a striking asymmetry between the PSP and SSP, just as one observes in the optical region. The PSP is seen over a phase interval of 0 p 30 , but the duration of the SSP is only 0?10. From Fig. 1b one can see that the velocity of the infalling material relative to the photosphere is twice that of the outflowing plasma. The non-photospheric Si II $\lambda 1264$ absorption that is seen outside of the defined shell phases is probably formed in the disk about the B primary. 

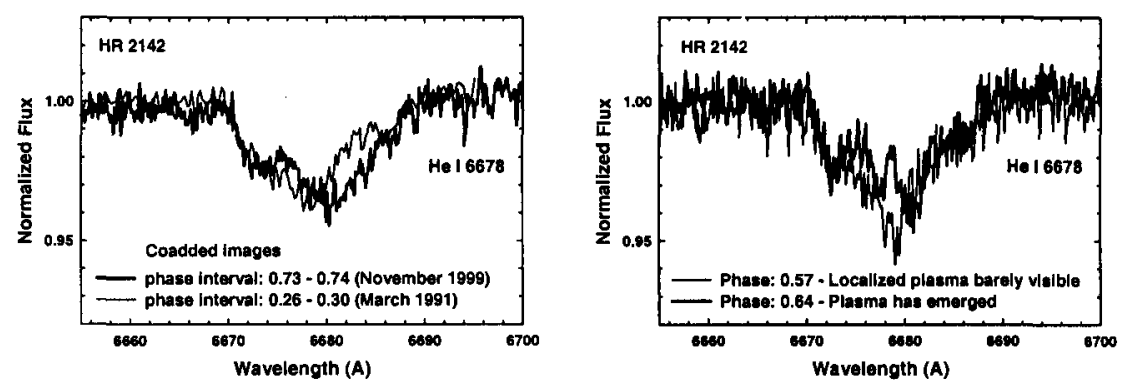

Figure 2. a. Left panel: Comparison between the profiles of $\mathrm{He} \mathrm{I}$ $\lambda 6678$ seen at quadrature. $b$. Right panel: The profiles observed just after inferior conjunction of the B star show the emergence of the plasma that yields the violet-shifted emission at phase 0.75 .

\section{The He I $\lambda 6678$ Emission Feature}

He I $\lambda 6678$ has proven to be a useful feature for studying the temporal and spatial variability in the $\sim 20,000 \mathrm{~K}$ plasma in B-type interacting binaries, as the emission from this line is intrinsically strong enough to be seen above the continuum of the B primary. In HR 2142 variable He I $\lambda 6678$ emission is clearly seen superimposed on the rotationally-broadened photospheric feature. From the display in Fig. 2a, it is apparent that the He emission is violet-shifted near phase 0.75 , the quadrature phase when we view the system toward its trailing hemisphere and the velocity of the B star is maximized. The reverse is observed at phase 0.25 . At first glance one might conclude that the plasma is associated with the secondary, because it appears to move in antiphase with the B star. Similar velocity behavior has been observed in the long-period Be binaries $\phi$ Per (Gies et al. 1993; Božić et al. 1995; Štefl, Hummel \& Rivinius 2000) and 59 Cyg (Harmanec et al. 2002) in which the presence of an $\mathrm{O}$ subdwarf has been confirmed (Gies et al. 1998) or suspected (Gies 2001) respectively. But the He I emission features in the latter binaries are considerably sharper and the motion displays a well-defined "S" curve symmetrically located about the conjunction points. There is no apparent symmetry in the emission profiles in HR 2142. The red-shifted emission near phase 0.25 is much more diffuse but there does appear to be an approximate symmetry in the centroid velocity $\left( \pm 130 \mathrm{~km} \mathrm{~s}^{-1}\right)$ that is close to the Keplerian velocity for material near the periphery of the disk. Now observations taken between inferior conjunction of the B star and phase 0.75 (Fig. 2b) indeed reveal that much of the He I-emitting plasma is occulted around phase 0.57 but has come into view by phase 0.64 . This places the emitting region around phase 0.05 , which also happens to be the phase interval where the SSP is observed. The plasma responsible for the variable $\mathrm{He}$ emission appears to be part of the SSP phenomenon. He I data taken during the SSP reveal violet-shifted shell-type absorption and diffuse emission between phases $0.02-0.06$. It is not uncommon to find a "localized plasma" between the components of a B-type interacting binary (Richards 1993; Richards et al. 2000; Peters 2001b) but its azimuth relative to the line-of-centers varies. 


\section{Discussion and Conclusions}

Our current perception of HR 2142 is illustrated in Fig. 3. The interpretation of the conspicuous mass infall/outflow must await the eventual detection of the secondary star but it appears reasonable to associate the "infall" region with a gas stream because the strength/velocity behavior of the infall lines and wide phase interval over which they are observed $(0.70<\phi<0.98)$ is virtually identical to what is seen in many B-type Algol systems of shorter period (Peters $2001 \mathrm{~b}$ ). However, the mass infall rate of $\sim 10^{-7} \mathrm{M}_{\odot} \mathrm{yr}^{-1}$ found from the analysis of the weak Si II (and Ti III) shell lines is too low to be responsible for the massive $\mathrm{H} \alpha$-emitting disk about the primary. The Be star in the HR 2142 system, as well as those in $\phi$ Per and 59 Cyg, was probably spun up during the rapid phase of mass transfer. The disks in these binaries are most likely a result of surface activity on the Be star and not current mass transfer.

An effort was made to recover the spectrum of a possible O-type subdwarf from the IUE database by using a Doppler tomography code (Bagnuolo et al. 1994), but convincing evidence for such a star was not found. From the depths of the most saturated features observed in the UV during the PSP and SSP, one finds an upper limit of $0.08 \mathrm{~F}_{\text {BStar }}$ for the brightness of a possible $\mathrm{O}$ subdwarf. This is half the relative flux found for the $\phi$ Per system (Gies et al. 1998).

The B primary appears to have a normal luminosity. From the Hipparcos parallax of 3.55 mas and an $E_{B-V}$ of 0.02 , one finds an $M_{V}$ of -2.10 . Adopting a $T_{\text {eff }}$ of $25,000 \mathrm{~K}$ (Peters 1983) one finds that the B star falls precisely on the $M_{V}-T_{\text {eff }}$ curve of Schönberner \& Harmanec (1995). Furthermore, the implied $M_{\text {bol }}$ places it on the zero age main sequence for $\sim 10.5 \mathrm{M}_{\odot}$ star according to the evolutionary tracks of Maeder (2001). Perhaps the secondary is not in thermal equilibrium (underluminous) or does not fill its Roche surface (cf. Peters 2001a). HR 2142 may represent an earlier evolutionary stage of $\phi$ Per or 59 Cyg. An assessment of the future of the HR 2142 system (SN Ib/c, II, WD pair, NS + WD) must necessarily await knowledge of the mass and nature of the secondary.

Acknowledgments. GJP is grateful for the support from NASA Grant NAG5-9124.

\section{References}

Bagnuolo, Jr., W. G., Gies, D. R., Hahula, M. E., Wiemker, R., \& Wiggs, M. S. 1994, ApJ, 423, 446

Božić, H., Harmanec, P., Horn, J., Koubský, Scholz, G., McDavid, D., Hubert, A. M., \& Hubert, H. 1995, A\&A, 304, 235

Gies, D. R. 2001, in The Influence of Binaries on Stellar Population Studies, ed.

D. Vanbeveren (Dordrecht: Kluwer), 95

Gies, D. R., Willis, C. Y., Penny, L. R., \& McDavid, D. 1993, PASP, 105, 281

Gies, D. R, Bagnuolo, Jr., W. G., Ferrara, E. C., Kaye, A. B., Thaller, M. L., Penny, L. R., \& Peters, G. J. 1998, ApJ, 493, 440

Harmanec, P., 1988, Bull. Astr. Inst. Czech, 39, 329

Harmanec, P., et al. 2002, A\&A, 387, 580 


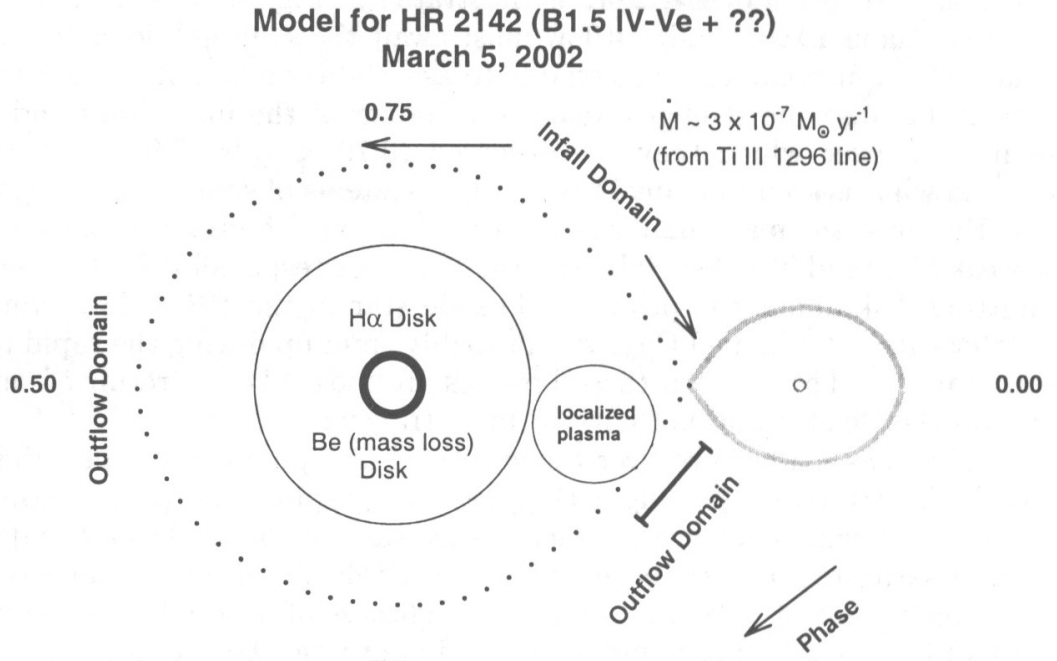

0.25

Figure 3. Our concept of the HR 2142 system in early 2002. The small circle on the left is the primary, while our current uncertainty in the nature of the secondary is indicated by the either/or star on the right. Possible secondaries include a Roche lobe-filling cool star or an O-type subdwarf. The dotted figure shows the Roche surface for the primary. The domains of infall/outflow and the localized plasma are indicated.

Maeder, A. 2001, in The Influence of Binaries on Stellar Population Studies, ed.

D. Vanbeveren (Dordrecht: Kluwer), 233

Peters, G. J. 1971, ApJ, 163, L107

Peters, G. J. 1972, PASP, 84, 334

Peters, G. J. 1976, in IAU Symp. 70, Be and Shell Stars, ed. A. Slettebak (Dordrecht: Reidel), 417

Peters, G. J. 1983,PASP, 95, 311

Peters, G. J. 2001a, Pub. Ast. Inst. Acad. Sci. Czech Republic, 89, 30

Peters, G. J. 2001b, in The Influence of Binaries on Stellar Population Studies, ed. D. Vanbeveren (Dordrecht: Kluwer), 79

Richards, M. T. 1993, ApJS, 86, 255

Richards, M. T., Koubský, P., Šimon, V., Peters, G. J., Hirata, R., Škoda, P., \& Masuda, S. 2000, ApJ, 531, 1003

Schönberner, D., \& Harmanec, P. 1995, A\&A, 294, 509

Štefl, S., Hummel, W., \& Rivinius, Th. 2000, A\&A, 358, 208

Waters, L. B. F. M., Coté, J., \& Pols, O. R. 1991, A\&A, 250, 437 